\title{
CHAF1B knockdown blocks migration in a hepatocellular carcinoma model
}

\author{
XIAODONG PENG $^{1 *}$, HUIYING FU $^{2 *}$, JIANJUN YIN $^{1}$ and QINGMEI ZHAO ${ }^{3}$ \\ ${ }^{1}$ Department of Oncology, The First Affiliated Hospital of Nanchang University, Nanchang, Jiangxi 330006; \\ ${ }^{2}$ Department of Oncology, The 94th Hospital of The People's Liberation Army, Nanchang, Jiangxi 330000; \\ ${ }^{3}$ Department of Oncology, Sichuan Mianyang 404 Hospital, Mianyang, Sichuan 361007, P.R. China
}

Received March 27, 2017; Accepted January 12, 2018

DOI: $10.3892 /$ or.2018.6437

\begin{abstract}
The roles and model of action of the chromatin assembly complex factor-1B (CHAF1B) gene in liver cancer have not been fully elucidated. The CHAF1B gene in human hepatocellular carcinoma cell line HUH-7 was knocked down using a lentivirus and the transfected cells were assayed for migration and invasion abilities and cell cycle arrest using the scratch wound healingand Transwell assays as well as flow cytometry, respectively. Cells transfected with an empty vector were used as the control. The expression of genes was profiled. Models were constructed using CHAF1B-knockdown cells and investigated for tumor growth and pathological changes. Our experiments revealed that the knockdown of the CHAF1 gene reduced the invasion and migration ability of HUH-7 cells. Gene expression profiling revealed that after knockdown, PSMB6, SLC30A7, SMC3, TWF2 and BLM genes had the most marked changes as compared with the control. Western blot and RT-PCR analyses revealed that following the knockdown of the CHAF1B gene, protein and mRNA levels of the PSMB6, SLC30A7 and SMC3 genes were significantly upregulated, while those of the BLM and TWF2 genes were significantly downregulated. In the HUH-7-knockdown cells, there were significantly fewer G0/G1 cells and more S1 cells as compared with the control (36.10 vs. $54.10 \%$ and 59.7 vs. $40.8 \%$, respectively), while the number of $\mathrm{G} 2 / \mathrm{M}$ cells was similar (4.20 vs. $5.10 \%)$. The volumes of the tumors were similar between those injected with the empty vector and
\end{abstract}

Correspondence to: Professor Jianjun Yin, Department of Oncology, The First Affiliated Hospital of Nanchang University, 17 Yongwaizheng Road, Nanchang, Jiangxi 330006, P.R. China

E-mail: yinjianjun01@126.com

Dr Qingmei Zhao, Department of Oncology, Sichuan Mianyang 404 Hospital, 56 Yuejin Road, Mianyang, Sichuan 361007, P.R. China

E-mail: zhaoqingmei29@163.com

*Contributed equally

Keywords: hepatocellular carcinoma, invasion, migration, gene expression control, but were significantly smaller in the knockdown models, suggesting that the knockdown of the CHAF1B gene inhibited tumor growth. H\&E staining revealed that tumors were developed in mice in all groups.

\section{Introduction}

Hepatocellular carcinoma (HCC) is one of the most common malignant tumors. It is the fifth most common cancer and ranks third in cancer-related deaths. In developing countries the incidence rate is even higher (1), and in developed countries the incidence rate is gradually increasing (2). Due to higher and earlier metastasis, HCC has a very poor prognosis with a 5-year survival of only $\sim 3-5 \%(3,4)$. Several options are available for HCC therapy, such as local treatment, surgical resection, targeted biological therapy, systemic chemotherapy and liver transplantation. However, the cure rate and overall survival have been unsatisfactory. Many cancer patients are unable to undergo operational therapy surgery due to poor liver function resulted resulting from chronic liver diseases (5). Therefore, there is an urgent need for early diagnosis and treatment of HCC. Identification of HCC-related genes is essential to have a better understating understanding of the pathogenic mechanisms in order to develop new diagnostic and therapeutic strategies.

Chromatin assembly factor $1(\mathrm{CAF}-1)$ is a trimer histone chaperone located in the nucleus, which mediates chromatin assembly after DNA replication and repair (6). CAF-1 is a heterotrimeric complex composed of three subunits p150, p60 and p48. It recruits histones $\mathrm{H} 3$ and $\mathrm{H} 4$ to DNA to facilitate nucleosome assembly, participates in the regulation of DNA repair and epigenetic changes in embryonic stem cellsas well as cell proliferation. It has been revealed to be an important histone chaperone in eukaryotic cells such as yeast and multicellular organisms (7). CAF1B is a p60 subunit of CAF-1, which is positively related to cell proliferation,and is located in the nucleolus during interphase and in the site of DNA replication in the $S$ phase (8). It has been proposed as a clinical marker to distinguish quiescent from proliferating cells (9). High expression of CAF-1 has been revealed to be associated with the invasion and metastasis of oral squamous cell carcinoma (10). High expression of CAF1B was demonstrated to be an indicator of poor prognosis in neuroblastoma (11). In the 
DNA synthesis phase CAF-1 is reversibly phosphorylated by Cyclin/Cdk2 (12). These findings indicated that the expression level of CAF-1 as well as CAF1B is closely related to tumor metastasis, invasion and poor prognosis $(11,13)$. Numerous studies have shown that CAF-1 controls specific chromatin recombination in the $\mathrm{S}$ phase to promote cell cycle progression and influences cell proliferation and apoptosis. It has been demonstrated that CAF-1 is essential for differentiation and proliferation of higher eukaryotic animal cells. Recently, CAF-1, particularly CHAF1B, was proposed as a new marker for tumor proliferation and prognosis and overexpression of CAF-1/p60 was used as a clinical marker for malignant tumor progression in some human malignant tumors $(14,15)$.

Previous studies have confirmed that CHAF1B is expressed in HCC cell lines Hep3B, HUH-7, HepG2 and SMMC-7721. Analysis of the expression data in the TCGA database revealed that the gene had higher mRNA abundance in a large number of HCC tissues than in the adjacent tissues. Therefore, we speculated that CHAF1B may be related to the biological behavior of HCC. In the present study, we knocked down the CHAF1B gene in HUH-7 cells using an RNAi lentiviral vector to investigate its effect on proliferation, apoptosis and the cell cycle of the cells. The findings would provide insight into the role of the gene in HCC, help in the identification of new signaling molecules for HCC research and validate its potential in HCC diagnosis and therapy.

\section{Materials and methods}

Cell line, reagents and equipment. Human HCC cell line HUH-7 was purchased from Yingniurui Biological Co. (Wuxi, Jiangsu, China). DMEM high glucose medium was obtained from Sijiqing Biotech (Hangzhou, China) (batch no. KGM12800S-500), DMSO was purchased from Amresco (Solon, OH, USA) (batch no. 302A0325), 0.25\% trypsin (containing EDTA) was purchased from Kaiji Biotech (Jiangsu, China) (batch no. 20160818), penicillin and streptomycin mix solution and GoldView I nucleic acid stain were obtained from Beijing Solarbio Science \& Technology Co., Ltd. (Beijing, China) (batch no. 20160909), fetal bovine serum (FBS) was purchased from BI Biotech (Beijing, China) (batch no. 1552680) and Transwell cells were obtained from Falcon (Atlanta, GA, USA). GoldStar Taq MasterMix and UltraSYBR Mixture were products from CWBiotech (Beijing, China). Primers were synthesized at General Biotech (Beijing, China). The ultra-sensitive chemiluminescence imaging system (ChemiDoc ${ }^{\mathrm{TM}} \mathrm{XRS}+$ ) and real-time PCR instrument CFX Connect ${ }^{\mathrm{TM}}$ were purchased by Bio-Rad Laboratories, Inc. (Hercules, CA, USA).

Experimental grouping. The cells were divided into the control that did not receive any treatment, the empty-vector group that was transfected with a vector without an insert andthe CHAF1B group that was transfected with CHAF1B.

Construction of lentivirus for CHAF1B knockdown. Interference sequences (upstream 5'-CACCGCCACTTAGAA GATGTGTATGCGAACATACACATCTTCTAAGTGGC and downstream 5'-AAAAGCCACTTAGAAGATGTGTAT GTTCGCATACACATCTTCTAAGTGGC) for CHAF1B were designed and synthesized and ligated to lentivirus vector pDS019_pL_shRNA_F. The lentivirus containing vector pDS019-PL-shRNA-GFP-homo-CHAF1B was packed and verified using a fluorescence microscope. The virus titer was determined using the $\mathrm{TCID}_{50}$ method and its expression was confirmed using western blot and PCR analyses. The virus was then amplified and purified using $\mathrm{CsC} 1$ gradient centrifugation. Cells without any treatment were used as the blank control, while the empty vector was used as a control.

Cell invasion and migration assays. Prior to the assessment of the invasion ability, the cells were infected with the CHAF1Bknockdown virus or control for $24 \mathrm{~h}$. Then $5 \times 10^{5}$ cells in serum-free medium were placed into the upper chamber of an insert ( $8 \mu \mathrm{m}$ pore size; BD Biosciences (San Jose, CA, USA). After $48 \mathrm{~h}$ of incubation, the cells remaining on the upper membrane were removed with cotton wool, whereas the cells that had migrated or invaded through the membrane were stained with $0.1 \%$ crystal violet in $25 \%$ methanol/PBS, imaged and counted under an inverted microscope (Life Technologies; Thermo Fisher Scientific, Inc., Waltham, MA, USA). The experiments were independently repeated three times.

For the assessment of migration ability, an in vitro scratch assay was used as previously reported (16).

Colony-formation ability assay. Cells $(0.4 \mathrm{ml})$ were inoculated into culture medium in the wells of a 96-plate (400 cells/well) in a total volume of $3 \mathrm{ml}$ and cultured for 2 to 3 weeks. The medium was refreshed every 3 days. Once the colonies became visible, they were fixed in methanol, and then washed and stained with Giemsa staining solution for $30 \mathrm{~min}$. The number of colonies were counted and the colony-forming ability was calculated. The experiments were repeated three times independently.

Flow cytometry. Cells were washed with pre-chilled PBS three times and fixed in $1 \mathrm{ml} 70 \%$ pre-chilled ethanol overnight at $4^{\circ} \mathrm{C}$ and washed again with PBS three times. The cells were re-suspended in $1 \mathrm{ml} \mathrm{PI} /$ Triton X-100 staining solution containing $0.2 \mathrm{mg}$ RNase A for $15 \mathrm{~min}$ at $37^{\circ} \mathrm{C}$ and then analyzed by flow cytometry. Cells $\left(2 \times 10^{4}\right)$ were assayed at each cell cycle.

Expression profiling and annotation. Total RNA was extracted using TRIzol reagent (Invitrogen; Thermo Fisher Scientific, Inc.) and an RNeasy Kit (Qiagen $\mathrm{GmbH}$, Hilden, Germany). The first strands of cDNA were synthesized using a cDNA synthesis kit (Invitrogen, Thermo Fisher Scientific, Inc.) and labelled using a monochromatic DNA labeling kit (Roche NimbleGen, Inc., Madison, WI, USA). The labeled cDNA was hybridized to GeneChip Human Gene 2.0 ST Array (Affymetrix; Thermo Fisher Scientific, Inc.) using a hybridization kit (Roche NimbleGen, Inc.) and the signals were scanned, with the GenePix 4000B chip scanner. The data were normalized using NimbleScan software (v. 2.0) and analyzed for differentially expressed genes using Agilent GeneSpring GX software (v. 11.5.1). Pathway analysis was performed against the latest KEGG (Kyoto Encyclopedia of Genes and Genomes) database. 
Real-time PCR.Total RNA was extracted from the cells using TRIzol reagent according to the supplier's instructions, reversely transcripted into cDNA and used for PCR assays using primers for CHAF1B as shown in Table I. PCR was carried out in a total volume of $20 \mu$ l containing $1 \mu \mathrm{l}$ of diluted and pre-amplified cDNA, $12.5 \mu \mathrm{l}$ of $2 \mathrm{X}$ UltraSYBR Mixture and $1 \mu \mathrm{l}$ of each fluorescence TaqMan probe using primers listed in Table I. The cycling conditions were $50^{\circ} \mathrm{C}$ for $2 \mathrm{~min}, 95^{\circ} \mathrm{C}$ for $3 \mathrm{~min}$ followed by 40 cycles, each one consisting of $15 \mathrm{sec}$ at $95^{\circ} \mathrm{C}$ and $30 \mathrm{sec}$ at $50.6^{\circ} \mathrm{C}$. The samples were run in triplicate and the mean value was calculated for each case.

The data were managed using the Applied Biosystems software RQ Manager v. 1.2.1 (Applied Biosystems, Foster City, CA, USA). The relative expression was calculated using the comparative $\mathrm{Ct}$ method and by obtaining the fold change value (2- $\left.{ }^{-\Delta \Delta \mathrm{Ct}}\right)$ according to a previously described protocol (17).

Western blot analysis. Cells were lysed using RIPA buffer (50 mM Tris, pH 7.2; $150 \mathrm{mM} \mathrm{NaCl} ; 1 \%$ Triton X-100; and $0.1 \%$ SDS) containing protease $(1: 100$; Roche Diagnostics, Indianapolis, IN, USA) and phosphatase (1:100; Sigma-Aldrich, St. Louis, MO, USA) inhibitors. The protein concentrations were determined using a bicinchoninic acid assay (Pierce; Thermo Fisher Scientific, Inc.). Proteins $(60 \mu \mathrm{g})$ were separated by SDS-PAGE and transferred (Bio-Rad Laboratories, Inc.) to PVDF membranes (EMD Millipore, Billerica, MA, USA). The membranes were incubated with rabbit polyclonal antibodies specific for SMC3 $(1: 2,000$; cat. no. ab9263) and $\beta$-actin (1:1,000; cat. no. ab8266; both from Abcam, Cambridge, MA, USA), PSMB6 (1:2,000; cat. no. A4053) TWF2 (1:2,000; cat. no. A5860) BLM (1:2,000; cat. no. A6535) and SLC30A7 (1:2,000; cat. no. A5172; all from ABclonal Biotech Co., Ltd., Woburn, MA, USA). The expression levels of these three proteins were standardized to human $\alpha$-actin using a mouse polyclonal anti- $\alpha$-actin antibody (1:1,000; cat. no. MAB1501R; EMD Millipore). Primary antibodies were detected using goat anti-rabbit or goat anti-mouse horseradish peroxidase (HRP)-conjugated secondary antibodies (1:1,000; AP189P; EMD Millipore). Immunoreactive bands were visualized using the ultra-sensitive chemiluminescence imaging system (ChemiDocXRS+) according to the manufacturer's instructions, and then quantified by densitometry using a ChemiGenius Gel Bio Imaging System (Syngene, Frederick, MD, USA).

Construction of HCC nude mouse models. Infected and control HUH-7 cells were digested, washed three times with PBS and re-suspended in PBS. Cells (200 $\mu \mathrm{l}$; 3x10\% $/$ mouse) were subcutaneously injected into nude mice. The animals were scarified three weeks later and the tumor tissues were collected for analysis.

$H \& E$ staining. Slides were dewaxed, dehydrated and stained with hematoxylin staining solution for $5 \mathrm{~min}$. The stained slides were soaked in hydrochloric acid alcohol for $30 \mathrm{sec}$, washed twice with PBS, briefly soaked in $0.5 \%$ ammonia for 1 to $2 \mathrm{~min}$. After being washed with PBS, the slides were counterstained with eosin for 2 min, washed 2 times with PBS, dehydrated, sealed with neutral balsam and images were captured under a
Table I. Primers for RT-PCR.

\begin{tabular}{lll}
\hline Gene & & \multicolumn{2}{c}{ Primer sequence } \\
\hline DNAJB1 & F & 3'-GATGGCTCTGATGTCATTTATC-5' \\
& R & 3'-GCCTTCTCCAGGAACTTTT-5' \\
SBNO1 & F & 3'-ATCCAGTGTTACTCCTCCTG-5' \\
& R & 3'-TGCTATCGTCCTTCCTTT-5' \\
BLM & F & 3'-TGAATCCAGAAACCAGCAC-5' \\
& R & 3'-AAGCAGTTCGTTCCCACA-5' \\
PSMB6 & F & 3'-CTGGGAAAGCCGAGAAGT-5' \\
& R & 3'-ATGCGGTCGTGAATAGGT-5' \\
SLC30A7 & F & 3'-TAGGCTTGATTTCCGACTC-5' \\
& R & 3'-CCGCTCTAACATACCCATA-5' \\
DDX3X & F & 3'-GGAGCGAATGCGTAAGGT-5' \\
& R & 3'-TTAGGGTGCGAAATGCTG-5' \\
TWF2 & F & 3-'AGGTTGTGATTGAGGACGAG-5' \\
& R & 3'-GGAGTTATCAGGCGACCAG-5' \\
SMC3 & F & 3'-TGTGATGAACCTCCTTGA-5' \\
& R & 3'-TCTGAGAATCTGGTGCTG-5' \\
TMEM259 & F & 3'-CGAGACGCCCACCAAAGT-5 \\
3-actin & R & 3'-CATCGTAGCCCAGGAACTCAT-5' \\
& F & 3'-CCTGTATGCCTCTGGTCG-5' \\
& R & 3'-GGCGTAACCCTCGTAGAT-5' \\
\hline
\end{tabular}

F, forward; R, reverse.

E400 Nikon microscope at x400 magnification (Nikon, Tokyo, Japan).

Statistical analysis. Statistical analyses were performed using SPSS 20 software (SPSS, Inc., Chicago, IL, USA). All experiments were repeated at least three times and performed in triplicate. The means were compared using the Student's t-test or one-way ANOVA with the corresponding post hoc test. $\mathrm{P} \leq 0.05$ was considered to indicate a statistically significant result.

\section{Results}

Transfection of HUH-7 cells with CHAFlB-silencing lentivirus. We examined the transfection efficiency by observing the intensity of GFP fluorescence $24 \mathrm{~h}$ after the transfection. Observations revealed that $>95 \%$ of cells were fluorescent after the cells were transfected with a lentivirus harboring CHAF1B-shRNA (Fig. 1A). No fluorescence was observed in the untransfected cells (data not shown). Western blot analysis revealed that $\mathrm{CHAF} 1 \mathrm{~B}$ was significantly downregulated at the protein level (Fig. 1B).

Invasion ability of HUH-7 cells after CHAF1B knockdown. We then examined the invasion ability of HUH-7 cells after the knockdown of the CHAF1B gene. The number of invaded cells was significantly lower after knockdown as compared to the control ( 16.33 vs. $42.13, \mathrm{P}<0.01)$, while the negative vector did not change the invasion ability (Fig. 2A), indicating that 


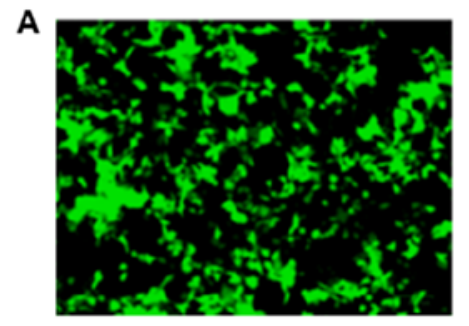

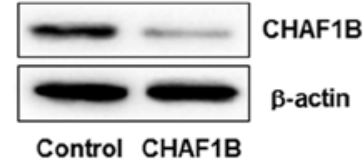

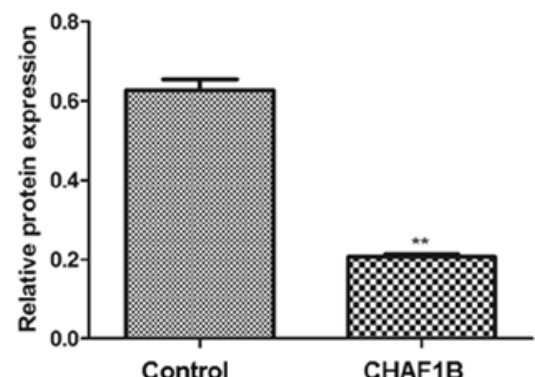

Figure 1. GFP fluorescence and expression of CHAF1B from HUH-7 cells transfected with a lentivirus harboring CHAF1B-shRNA. (A) GFP fluorescence and (B) the protein level of CHAF1B. ${ }^{* *} \mathrm{P}<0.01$.

A

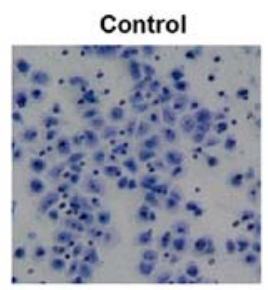
Negative vector

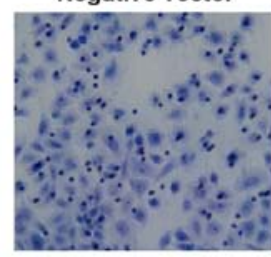

B

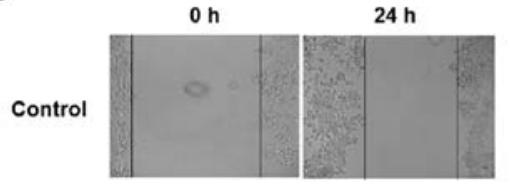

CHAF1B

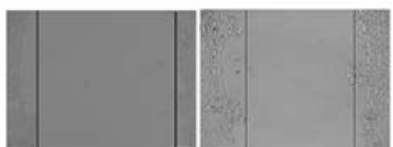

\section{CHAFB KD}

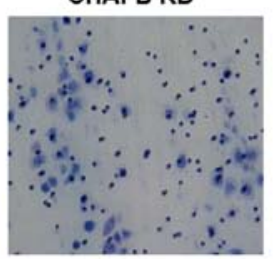

C

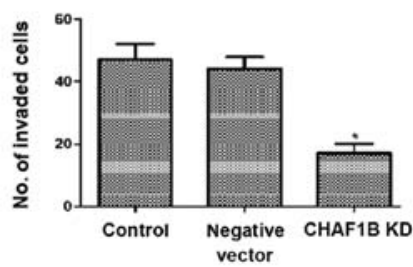

vector

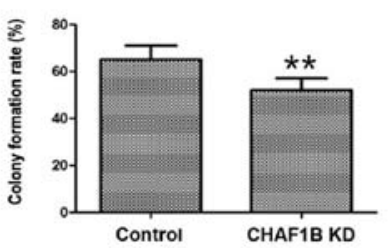

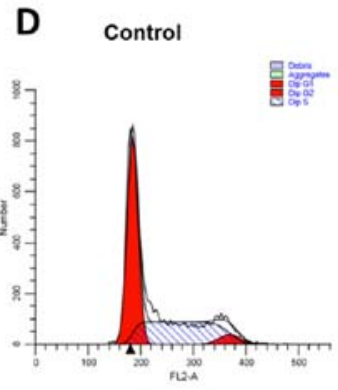

G0/G1: $54.10 \%$

S: $40.80 \%$

$\mathrm{G} 2 / \mathrm{M}: \quad 4.20 \%$
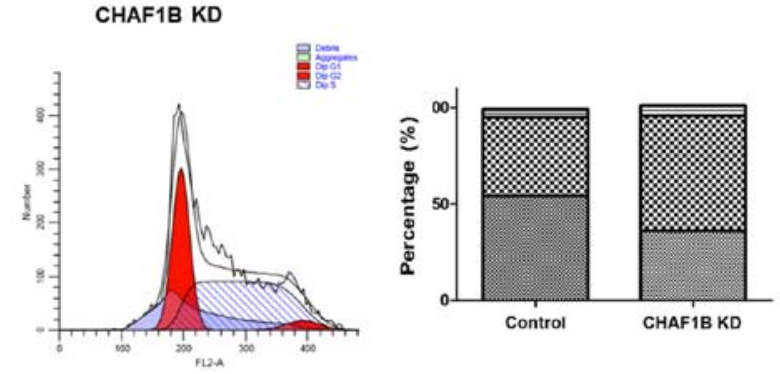

GO/G1: $36.10 \%$

S: $59.70 \%$

$\mathrm{G} 2 / \mathrm{M}: 5.10 \%$

Figure 2. Invasion, migration, colony-forming ability and the cell cycle of HUH-7 cells after the knockdown of CHAF1B. (A) Right panel, microimages of the Transwell assay; left panel, the number of invaded cells. (B) Microimages of the scratch assay. (C) Colony formation rate. (D) Right panel, flow cytometric results; left panel, the percentage of cells in different cell phases. ${ }^{*} \mathrm{P}<0.05$ and ${ }^{* *} \mathrm{P}<0.01$ vs. the control, respectively.

the downregulation of $\mathrm{CHAF} 1 \mathrm{~B}$ expression reduces the invasion ability of HUH-7 cells, suggesting that it may be used as a new approach for HCC therapy.

Migration and colony-forming ability of $\mathrm{HUH}-7$ cells after CHAF1B knockdown. We next investigated the effect of CHAF1B knockdown on migration and colony-forming ability of HUH-7 cells. The scratch assays revealed that compared with the control, CHAF1B knockdown significantly reduced the migration distance of HUH-7 cells (98.6 $\pm 3.29 \mu \mathrm{m}$ vs. $41.2 \pm 2.59 \mu \mathrm{m}, \mathrm{P}<0.01)$ (Fig. 2B). We also compared the colony formation ability as a measure of tumor-forming ability. The results revealed that $\mathrm{CHAF} 1 \mathrm{~B}$ knockdown significantly reduced the colony formation rate as compared with the control (Fig. $2 \mathrm{C}, \mathrm{P}<0.01$ ). These findings revealed that silencing of $\mathrm{CHAF} 1 \mathrm{~B}$ may reduce the tumorigenic ability of HUH-7 cells and inhibit tumor occurrence and development. 

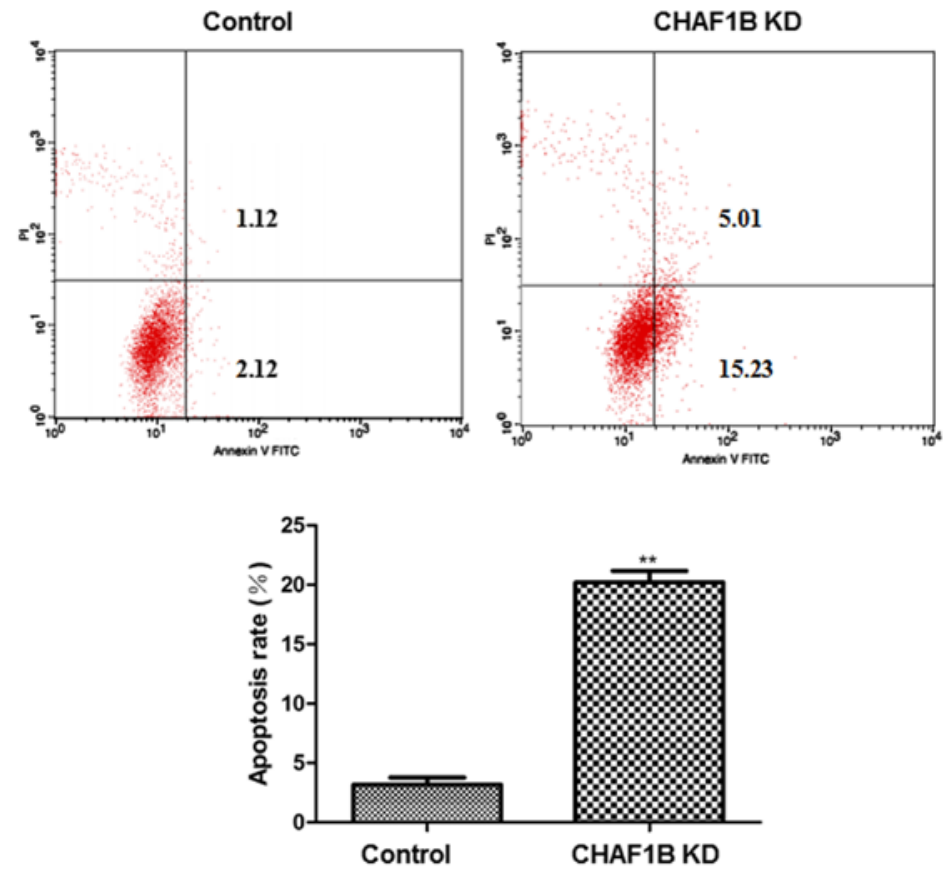

Figure 3. Apoptosis of HUH-7 cells after CHAF1B knockdown. Upper panel, flow cytometric results; lower panel, percentage of apoptotic cells. ${ }^{* *} \mathrm{P}<0.01$.

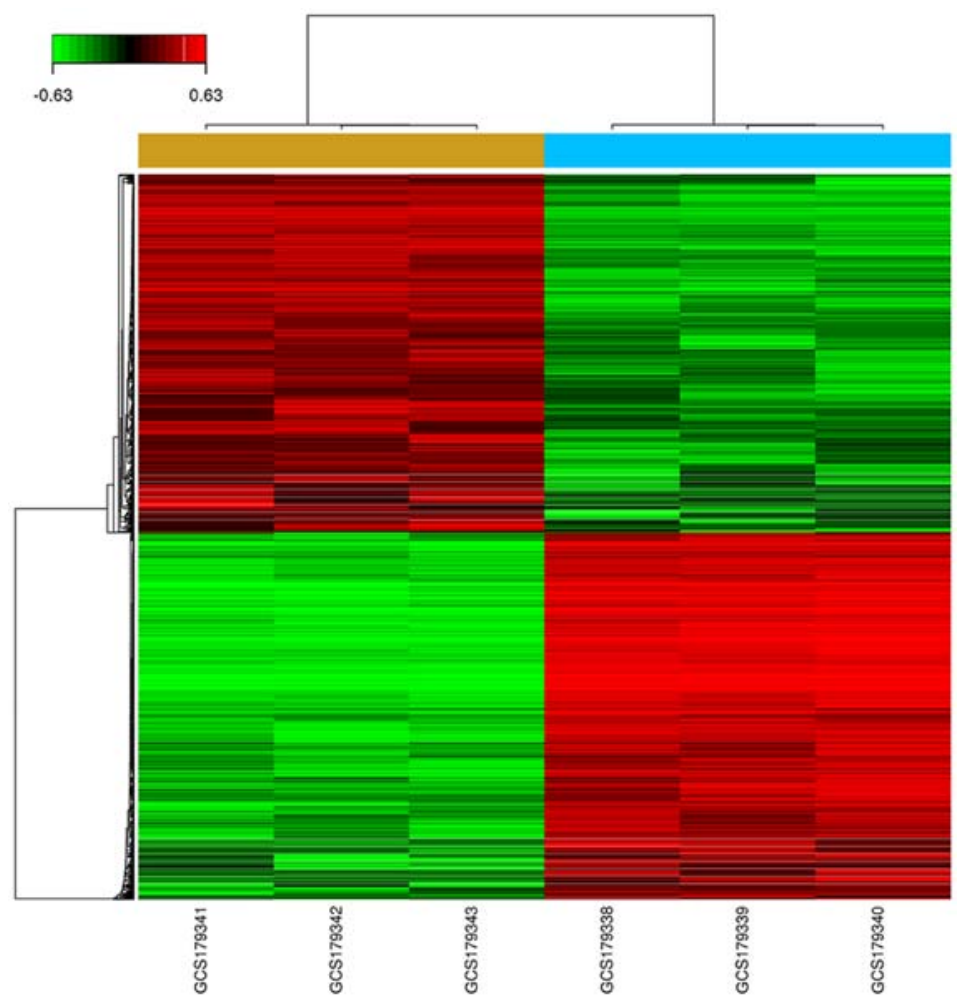

Figure 4. Expression profiling of genes in HUH-7 cells after CHAF1B knockdown.

Effect of CHAF1B knockdown on the cell cycle of HUH-7 cells. Flow cytometric analysis revealed that there were significantly less HUH-7 cells in the $G_{0} / G_{1}$ phase and more in the $S$ phase after CHAF1B knockdown (36.10 and 59.7 vs. 54.10 and $40.8 \%$ ). However, the percentage of cells at the G2/M phase were similar (4.20 vs. $5.10 \%)$, indicating that knockdown of the CHAF1B gene reduced the number of cells in the $\mathrm{G} 1$ phase and increased the cells in the $S$ phase, resulting in changes in the distribution of cells in different phases (Fig. 2D).
Effect of CHAF1B knockdown on the apoptosis of HUH-7 cells. Flow cytometric analysis revealed that following CHAF1B knockdown, HUH-7 cells had a significantly higher apoptosis rate as compared with the control (Fig. 3).

Expression of downstream genes in HUH-7 cells after CHAF1B knockdown. We profiled the expression of genes using the gene chip analysis. Several genes were found differentially expressed in the knockdown cells as compared with the control (Fig. 4), 

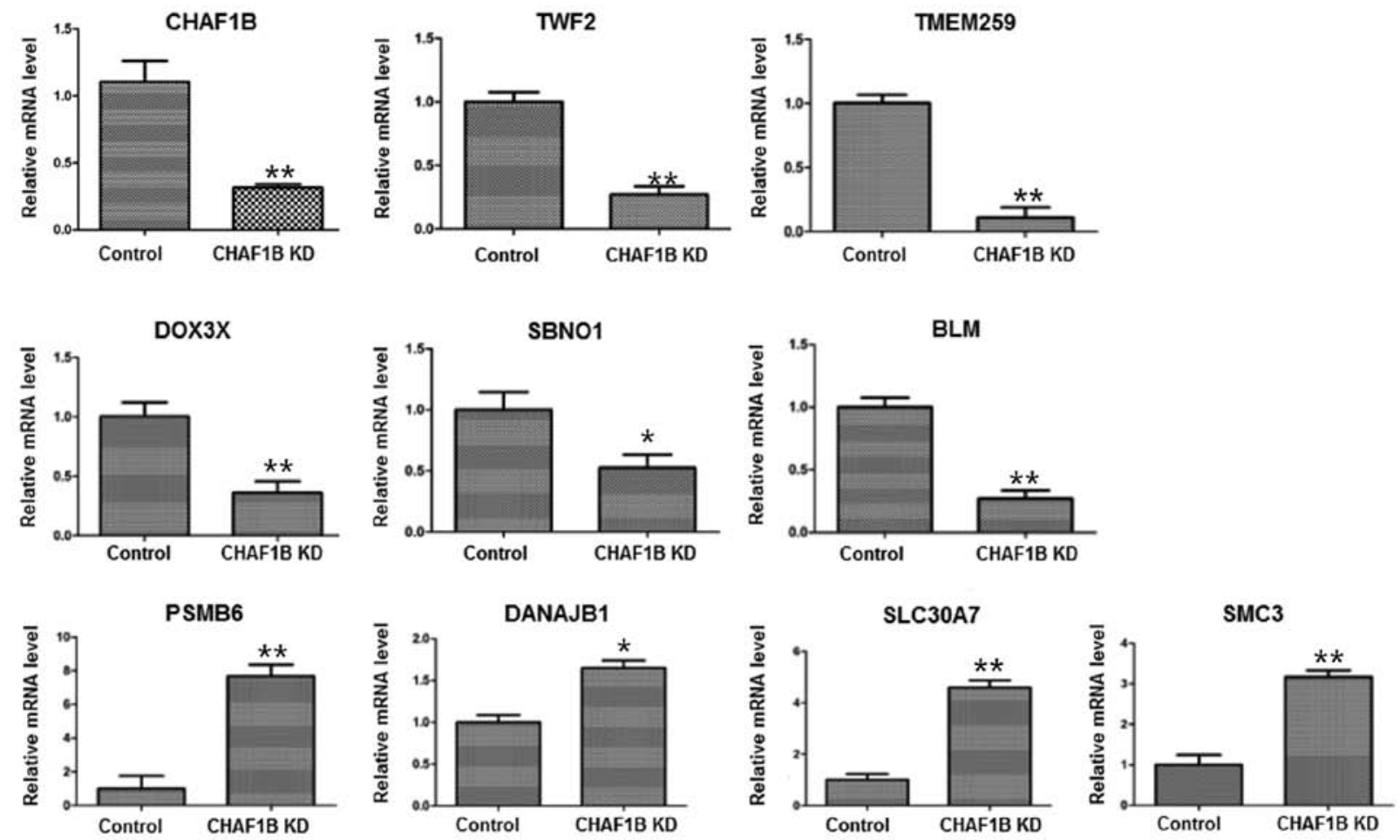

Figure 5. mRNA levels of downstream genes in HUH-7 cells after CHAF1B knockdown. (A) Expression profiling. ${ }^{*} \mathrm{P}<0.05$ and ${ }^{* *} \mathrm{P}<0.01$ vs. the control, respectively.
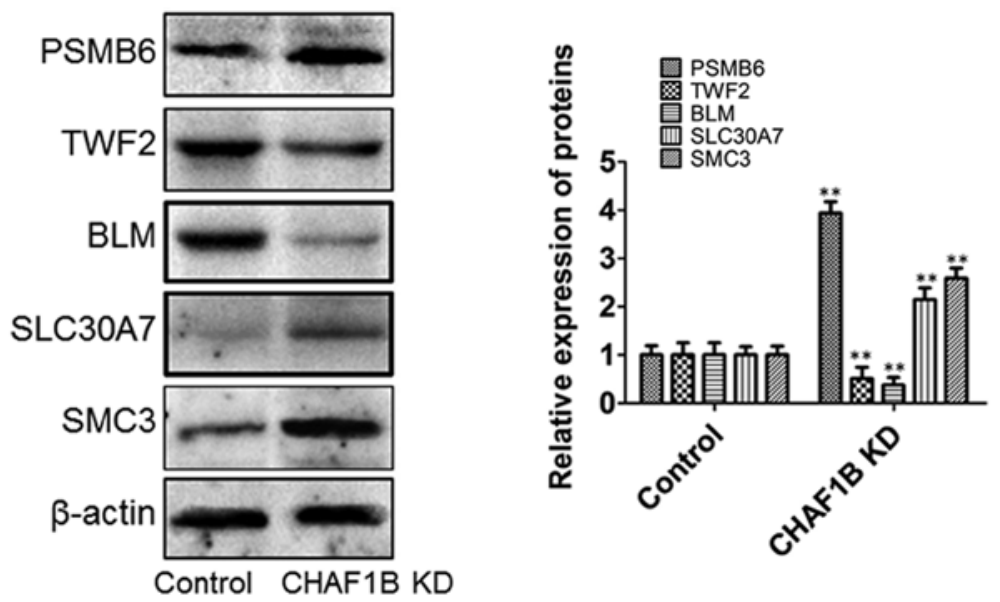

Figure 6. Western blot analyses of downstream genes in HUH-7 cells after CHAF1B knockdown. Right panel, representativewestern blots; left panel, relative protein content. ${ }^{*} \mathrm{P}<0.05$ and ${ }^{* *} \mathrm{P}<0.01$ vs. the control, respectively.

among them, the expression levels of PSMB6, SLC30A7, SMC3, TWF2 and BLM were the most markedlyaltered. RT-PCR results revealed that the mRNA levels of TWF2, TMEM259, DDX3X, SBNO1 and BLM were downregulated while the levels of PSMB6, DNAJB1, SLC30A7 and SMC3 were upregulated after CHAF1B knockdown (Fig. 5). Western blot analyses revealed that the protein levels of PSMB6, SLC30A7 and SMC3 were significantly increased and the levels of BLM and TWF2 were significantly decreased following CHAF1B knockdown (Fig. 6).

Effect of CHAF1B knockdown on tumor growth. We then compared the growth of tumors in the mouse models prepared using CHAF1B-knockdown HUH-7 cells. Twenty-eight days after the injections, the volume and tumor formation rate were similar between the control and the empty vector. However, these figures were significantly lower in mice injected with CHAF1B knockdown-cells as compared with the control (Fig. 7A). H\&E staining further confirmed that tumors were present in rats in all groups (Fig. 7B). Western blot analyses revealed that CHAF1B was significantly lower after CHAF1B-knockdown (Fig. 8A) and TUNEL assay revealed that there were more apoptotic cells in the CHAF1B-knockdown HUH-7 cells (Fig. 8B). These findings demonstrated that CHAF1B knockdown reduced the tumorigenicity of HUH-7 cells. 


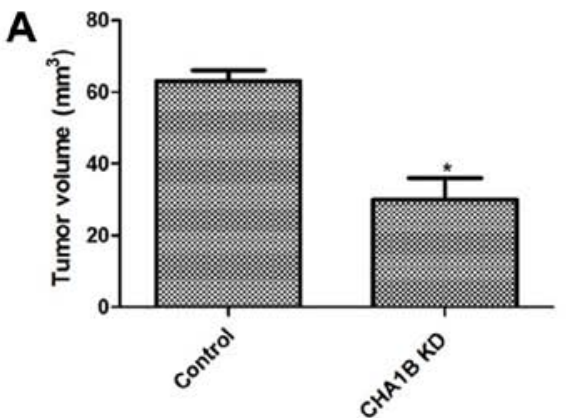

B
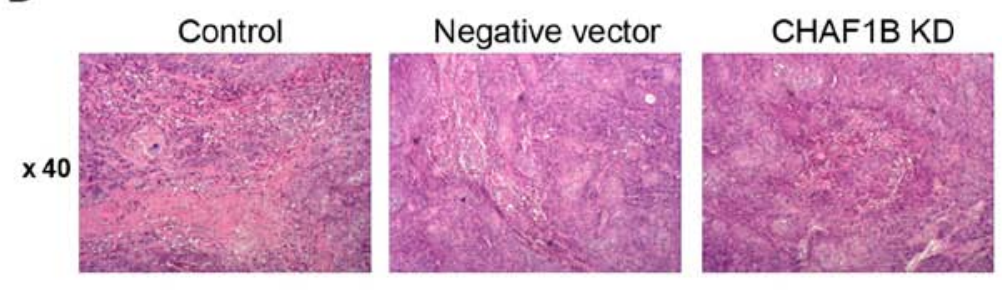

Figure 7. Development of the tumors and pathological changes in mice injected with CHAF1B-knockdown HUH-7 cells. (A) Tumor volume and (B) H\&E staining of the tumor tissues. " $\mathrm{P}<0.05$ vs. control.
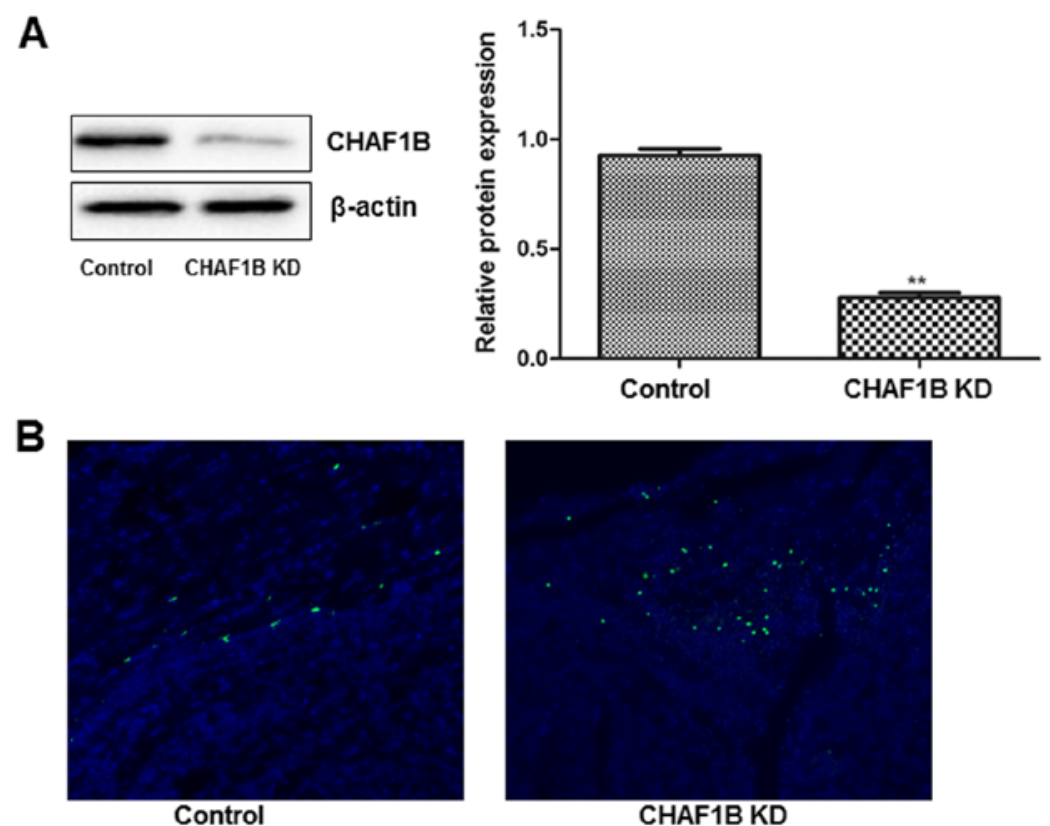

Figure 8. CHAF1B expression and HUH-7 cell apoptosis after CHAF1B knockdown. (A) Left panel, representative western blot; right panel, relative CHAF1B level. (B) TUNEL assay. ${ }^{* *} \mathrm{P}<0.01$.

\section{Discussion}

Chromatin assembly factors (CAFs) have subunits of different sizes (150 kDa, CAF-1-p150 or CHAF1a), (60 kDa, CAF-1-p60 or CHAF1B) and (48 kDa, CAF-1-p48 or RbAp48, p48) (18). Verreault et al (19) purified the complex and revealed that CHAF1B can reposition nucleosomes, and change the position and structure of nucleosomes to regulate the level of chromatin during DNA replication, transcription, repair and recombination (20). They determined that transcriptional factors (TFs) and CAFs bind to specific sites in the promoter to change the position and structure of nucleosomes and sensitize chromatin to ribozymes. The synthesis of DNA is the basis for nucleosome assembly and plays a key role in maintaining genome stability. During the replication of DNA, the synthesis of CAF-1 in histones $\mathrm{H} 3$ and $\mathrm{H} 4$ is turned off directly in many tissues, and the large subunit (CAF-1-P150, CHAF1a) and small subunit (RbAp48, P48) of CAF-1 have a variety of functions, for instance, as histone chaperones (21).

In recent years, CAF1B has been demonstrated to be highly expressed in a variety of malignant tumors, and has been identified as a marker of poor prognosis in cancer patients $(7,10)$. The expression level of CHAF1B has been demonstrated to be related to tumor invasiveness (11). It has been revealed that from radial growth in the early stage to pituitary growth, the expression level of CAF1B is significantly elevated, and the level also increases during the transition from benign nevus to malignant melanoma (21). Polo et al revealed that in breast, cervical and endometrial cancer as well as in renal cell carcinoma, higher expression of CAF-1 was related to histological grading and may be used as an independent factor of poor prognosis (22). Our experimental results also revealed that knockdown of the CHAF1B gene reduced the invasiveness, migration and colony-forming ability of HCC cells, further confirming that CHAF1B is involved in HCC biology.

CHAF1B may play a role in predicting therapeutic efficacy and monitoring drug response (22). Inhibition of this protein in invasive tumors can lead to tumor cell death and therefore it may become a new therapeutic target (23). CAF-1 is a p48 complex composed of three subunits p150, p60, and p48 $(21,24-26)$. Its compositions and functions are highly conserved in genome 
replication (27). The primary role of CAF-1 in DNA replication is to facilitate the first step in assembling nucleosomes to the newly synthesized DNA $(9,28,29)$. At any time in the cell cycle, DNA damage could lead to the initiation of DNA damage response (DDR). In eukaryotic tissues, DDR coordinates DNA damage repair and repair of altered chromatin (30). For example, CAF-1 may recruit a large number of damage-repairing proteins to the damaged chromatin regions as histone chaperones and/or the protein platform to participate in DNA and chromatin damage repair (9,31-33). Recent studies also demonstrated that CAF-1 may serve as a protein platform of epigenetics to inhibit chromatin markers or signal transduction of active histone, which are important for normal cell activities $(34,35)$. In addition, numerous studies have revealed that CAF-1 promoted cell cycle progression by adjusting the specific chromatin reorganization in the $\mathrm{S}$ phase to affect cell proliferation (34).

In addition, CAF-1 has been demonstrated to be associated with human diseases. The main function of the $\mathrm{p} 48$ subunit is to support the role of retinoblastoma protein RbAp48 in the inhibition of cell growth. p150 is directly related to proliferating cell nuclear antigen (PCNA), and actively participates in the DNA repair process during reproduction (28). The CAF1B subunits are highly expressed in breast, oral, tongue, prostate and salivary gland cancer, as well as in skin melanoma and other malignant tumors and downregulation of p150 in tongue cancer and head and neck cancer is an indicator for poor prognosis (36). The present study also demonstrated that the knockdown of the CHAF1B gene significantly inhibited cell proliferation and promoted apoptosis.

Collectively, CAF1B elevation was associated with poor prognosis in $\mathrm{HCC}$, and related to tumor stage, grade and distant metastasis. Therefore, understanding the expression level of CAF1B is helpful for the diagnosis and treatment of HCC, although more studies are warranted to elucidate the specific mechanism. Our study demonstrated that downregulation of CHAF1B inhibited the proliferation of HCC cells and induced apoptosis. These findings provided insight into the involvement of the CHAF1B gene in tumor signal transduction pathways and indicates the possible use of the gene for clinical diagnosis and targeted treatment.

\section{Acknowledgements}

This study was supported bythe Department of Science and Technology of Jiangxi Province (grant no. 20152ACG70020).

\section{Competing interests}

The authors declare that they have no competing interests.

\section{References}

1. Niu J, Lin Y, Guo Z, Niu M and Su C: The epidemiological investigation on the risk factors of hepatocellular carcinoma: A case-control study in southeast china. Medicine 95: e2758, 2010.

2. El-Serag HB and Mason AC: Rising incidence of hepatocellular carcinoma in the United States. N Engl J Med 340: 745-750, 1999.

3. Llovet JM, Burroughs A and Bruix J: Hepatocellular carcinoma. Lancet 362: 1907-1917, 2003.
4. Yang XD, Pan LH, Wang L, Ke Y, Cao J, Yang C, Zhong JH, Luo W, Guo J and Li LQ: Systematic review of single large and/or multinodular hepatocellular carcinoma: Surgical resection improves survival. Asian Pac J Cancer Prev 16: 5541-5547, 2015.

5. Lee CW, Tsai HI, Sung CM, Chen CW, Huang SW, Jeng WJ, Wu TH, Chan KM, Yu MC, Lee WC and Chen MF: Risk factors for early mortality after hepatectomy for hepatocellular carcinoma. Medicine 95: e5028, 2016.

6. De Koning L, Corpet A, Haber JE and Almouzni G: Histone chaperones: An escort network regulating histone traffic. Nat Struct Mol Biol 14: 997-1007, 2007.

7. Xu M, Jia Y, Liu Z, Ding L, Tian R, Gu H, Wang Y, Zhang H, Tu K and Liu Q: Chromatin assembly factor 1, subunit A (P150) facilitates cell proliferation in human hepatocellular carcinoma. Onco Targets Ther 9: 4023-4035, 2016.

8. Yu Z, Liu J, Deng WM and Jiao R: Histone chaperone CAF-1: Essential roles in multi-cellular organism development. Cell Mol Life Sci 72: 327-337, 2015.

9. Polo SE, Roche D and Almouzni G: New histone incorporation marks sites of UV repair in human cells. Cell 127: 481-493, 2006.

10. Mascolo M, Ilardi G, Merolla F, Russo D, Vecchione ML, de Rosa G and Staibano S: Tissue microarray-based evaluation of chromatin assembly factor-1 (CAF-1)/p60 as tumour prognostic marker. Int J Mol Sci 13: 11044-11062, 2012.

11. Staibano S, Mascolo M, Rocco A, Lo Muzio L, Ilardi G, Siano M, Pannone G, Vecchione ML, Nugnes L, Califano L, et al: The proliferation marker chromatin assembly factor- 1 is of clinical value in predicting the biological behaviour of salivary gland tumours. Oncol Rep 25: 13-22, 2011.

12. Jeffery DC, Kakusho N, You Z, Gharib M, Wyse B, Drury E, Weinreich M, Thibault P, Verreault A, Masai H and Yankulov K: CDC28 phosphorylates Cac1p and regulates the association of chromatin assembly factor I with chromatin. Cell Cycle 14: 74-85, 2015.

13. Wu Z, Cui F, Yu F, Peng X, Jiang T, Chen D, Lu S, Tang H and Peng Z: Up-regulation of CHAF1A, a poor prognostic factor, facilitates cell proliferation of colon cancer. Biochem Biophys Res Commun 449: 208-215, 2014.

14. Mascolo M, Ayala F, Ilardi G, Balato A and Lembo S: Chromatin assembly factor- $1 / \mathrm{p} 60$ overexpression: A potential index of psoriasis severity. Eur J Dermatol 24: 509-511, 2014.

15. Sarkar D, Leung EY, Baguley BC, Finlay GJ and AskarianAmiri ME: Epigenetic regulation in human melanoma: Past and future. Epigenetics 10: 103-121, 2015.

16. Liang CC, Park AY and Guan JL: In vitro scratch assay: A convenient and inexpensive method for analysis of cell migration in vitro. Nat Protoc 2: 329-333, 2007.

17. Livak KJ and Schmittgen TD: Analysis of relative gene expression data using real-time quantitative PCR and the $2^{-\Delta \Delta C \mathrm{~T}}$ method. Methods 25: 402-408, 2001.

18. Volk A and Crispino JD: The role of the chromatin assembly complex (CAF-1) and its p60 subunit (CHAF1b) in homeostasis and disease. Biochim Biophys Acta 1849: 979-986, 2015.

19. Verreault N, Da Costa D, Marchand A, Ireland K, Banack H, Dritsa $M$ and Khalife S: PTSD following childbirth: Prospective study of incidence and risk factors incanadian women. J PsychosomRes 73: 257-266, 2012.

20. Cheloufi S, Elling U, Hopfgartner B, Jung YL, Murn J, Ninova M, Hubmann M, Badeaux AI, Euong Ang C, Tenen D, et al: The histone chaperone CAF-1 safeguards somatic cell identity. Nature 528: 218-224, 2015.

21. Kaufman PD, Kobayashi R, Kessler N and Stillman B: The p150 and p60 subunits of chromatin assembly factor I: A molecular link between newly synthesized histones and DNA replication. Cell 81: 1105-1114, 1995.

22. Polo SE, Theocharis SE, Grandin L, Gambotti L, Antoni G, Savignoni A, Asselain B, Patsouris E and Almouzni G: Clinical significance and prognostic value of chromatin assembly factor-1 overexpression in human solid tumours. Histopathology 57: 716-724, 2010.

23. Mascolo M, Ilardi G, Romano MF, Celetti A, Siano M, Romano S, Luise C,Merolla F, Rocco A, Vecchione ML, et al: Overexpression of chromatin assembly factor-1 p60, poly(ADP-ribose) polymerase 1 and nestin predicts metastasizing behaviour of oral cancer. Histopathology 61: 1089-1105, 2012.

24. Kim D, Setiaputra D, Jung T, Chung J, Leitner A, Yoon J, Aebersold R, Hebert H, Yip CK and Song JJ: Molecular architecture of yeast chromatin assembly factor 1. Sci Rep 6: 26702, 2016. 
25. Smith S and Stillman B: Purification and characterization of CAF-I, a human cell factor required for chromatin assembly during DNA replication in vitro. Cell 58: 15-25, 1989.

26. Zhou H, Madden BJ, Muddiman DC and Zhang Z: Chromatin assembly factor 1 interacts with histone H3 methylated at lysine 79 in the processes of epigenetic silencing and DNA repair. Biochemistry 45: 2852-2861, 2006.

27. Shibahara K and Stillman B: Replication-dependent marking of DNA by PCNA facilitates CAF-1-coupled inheritance of chromatin. Cell 96: 575-585, 1999.

28. Moggs JG, Grandi P, Quivy JP, Jónsson ZO, Hübscher U, Becker PB and Almouzni G: A CAF-1-PCNA-mediated chromatin assembly pathway triggered by sensing DNA damage. Mol Cell Biol 20: 1206-1218, 2000.

29. Krude T: Chromatin assembly factor $1(\mathrm{CAF}-1)$ colocalizes with replication foci in HeLa cell nuclei. Exp Cell Res 220: 304-311, 1995.

30. Li GM: New insights and challenges in mismatch repair: Getting over the chromatin hurdle. DNA Repair 19: 48-54, 2014.

31. Adam S, Polo SE and Almouzni G: Transcription recovery after DNA damage requires chromatin priming by the $\mathrm{H} 3.3$ histone chaperone HIRA. Cell 155: 94-106, 2013.
32. Baldeyron C, Soria G, Roche D, Cook AJ and Almouzni G: HPlalpha recruitment to DNA damage by $150 \mathrm{CAF}-1$ promotes homologous recombination repair. J Cell Biol 193: 81-95, 2011.

33. Yu Z, Wu H, Chen H, Wang R, Liang X, Liu J, Li C, Deng WM and Jiao R: CAF-1 promotes notch signaling through epigenetic control of target gene expression during drosophila development. Development 140: 3635-3644, 2013.

34. Huang H, Yu Z, Zhang S, Liang X, Chen J, Li C, Ma J and Jiao R: Drosophila CAF-1 regulates HP1-mediated epigenetic silencing and pericentric heterochromatin stability. J Cell Sci 123: 2853-2861, 2010.

35. Wen P, Quan Z and Xi R: The biological function of the WD40 repeat-containing protein p55/Caf1 in drosophila. Dev Dyn 241: 455-464, 2012.

36. Staibano S, Mignogna C, Lo Muzio L, Mascolo M, Salvatore G, Di Benedetto M, Califano L, Rubini C and De Rosa G: Chromatin assembly factor-1 (CAF-1)-mediated regulation of cell proliferation and DNA repair: A link with the biological behaviour of squamous cell carcinoma of the tongue? Histopathology 50: 911-919, 2007. 